\title{
DNA Microarray Analysis
}

National Cancer Institute

\section{Source}

National Cancer Institute. DNA Microarray Analysis. NCI Thesaurus. Code C25922.

This term describes an experiment in which probes representing genes that one wants to study are affixed to a substrate (e.g., a glass slide) and then exposed to target molecules (sometimes referred to as the sample). The level of hybridization between a specific probe and a target (typically indicated through fluorescence and measured through image analysis) indicates the level of the gene corresponding to that probe in a test solution. 